\section{UK trainee experience in interstitial lung disease: results from a British Thoracic Society survey}

\section{ABSTRACT}

Interstitial lung disease (ILD) is a growing field of respiratory medicine in which novel therapies are emerging. It is important that trainees gain competence and confidence in this area. To explore the training experiences of specialty trainees, we conducted a survey of their practical experience and confidence in diagnosing and managing ILD.

Interstitial lung disease (ILD) is a growing field of respiratory medicine in which novel therapies are emerging. The provision of services varies across the country, as evidenced by the British Thoracic Society (BTS) ILD survey in 2011. ${ }^{1}$ It is important that trainees gain competence and confidence in this area. To explore the training experiences of specialty trainees, we conducted a survey of their practical experience and confidence in diagnosing and managing ILD. Please see online supplementary files for questionnaire content and survey methodology.

\section{RESULTS}

There were 104 respondents out of a possible 574 . There were no gross variations in the seniority or geography of respondents. The majority (67\%) were working in teaching hospitals at the time of the survey.

A significant proportion of respondents (33\%) were not expecting any subspecialty clinics in ILD during the course of their training. Forty-two per cent of trainees expect to spend 3 months or less attending specialist clinics. While the majority of trainees will experience ILD multidisciplinary teams (MDTs), their attendance is not guaranteed, with $45 \%$ expecting to attend fewer than half of MDTs during their period in specialist ILD hospitals.

The majority of trainees are trained in performing both bronchoalveolar lavage
(BAL) for cell differential analysis (73\%) and transbronchial biopsies (84\%); however, only $48 \%$ are confident of performing a transbronchial biopsy.

While $94 \%$ and $78 \%$ of respondents are confident interpreting gas transfer and 6-minute walk tests, respectively, the confidence interpreting more specialist tests is much lower (cardiopulmonary exercise testing 21\%, cell differential from BAL $38 \%)$.

The self-rated knowledge in a range of subject areas was assessed and demonstrated that most areas were moderately well understood; however, knowledge of the rarer diseases was rated lower.

The majority of trainees (54\%) felt that their ILD training was inadequate preparation for the specialty certificate exam. Ninety-four per cent would value a BTS short course on ILD to improve their knowledge and confidence.

\section{DISCUSSION}

This survey highlights the areas where there are clear opportunities to enhance the training of registrars in ILD. There are a significant number without subspecialty exposure during their training, and attendance at ILD MDTs appears sporadic. It is notable that some of the data conflict with previous BTS surveys in this area, and self-selection bias in our population is possible, skewed towards more interested trainees.

While most trainees are trained in performing necessary procedures, their confidence interpreting results of common investigations in ILD is low. To give evidence of training and competence in this area, deaneries may wish to consider requesting logbook evidence of procedures related to ILD (BAL for cell differential, transbronchial biopsies), in addition to evidence of ILD MDT attendance.

Where possible, it would seem sensible for deaneries to ensure rotation through hospitals with expertise in the subspecialist management of ILDs with a clear desire to optimally train registrars. This is especially pertinent given the recent advances in the management of ILD, including new antifibrotic medications such as pirfenidone and the immunosuppressive approaches to connective tissue disease-related ILD. These treatments are, and will probably remain, confined to specialist centres, thereby limiting trainee exposure.

\section{Charles Sharp, ${ }^{1}$ Toby M Maher, ${ }^{2}$ Sally Welham, ${ }^{3}$} Michael Gibbons ${ }^{4}$

${ }^{1}$ Academic Respiratory Group, Learning and Research, Southmead Hospital, Bristol, UK

${ }^{2}$ Interstitial Lung Disease Unit, Royal Brompton Hospital, London, UK

${ }^{3}$ British Thoracic Society, London, UK

${ }^{4}$ Respiratory Department, Royal Devon and Exeter NHS Foundation Trust, Exeter, UK

Correspondence to Dr Charles Sharp, Academic Respiratory Group, Learning and Research, Southmead Hospital, Bristol BS10 5NB, UK; Charles.sharp@doctors.org.uk

Contributors CS conceived the work and formulated the survey with MG in consultation with TMM and SW. CS compiled and analysed data. The manuscript was written by all authors.

Competing interests None.

Provenance and peer review Not commissioned; internally peer reviewed.

Data sharing statement Further data are available to any interested parties from the corresponding author.

- Additional material is published online only. To view please visit the journal online (http://dx.doi.org/10. 1136/thoraxjnl-2014-206397).

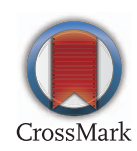

To cite Sharp C, Maher TM, Welham S, et al. Thorax 2015;70:183.

Received 2 October 2014

Revised 9 October 2014

Accepted 9 October 2014

Published Online First 28 October 2014

Thorax 2015;70:183.

doi:10.1136/thoraxjnl-2014-206397

\section{REFERENCE}

1 Dempsey OJ, Welham S, Hirani N. BTS National Interstitial Lung Diseases (ILD) Survey 2010-2011. Thorax 2011;66(Suppl 4):A102. 